Virginia Commonwealth University

vCU Scholars Compass

Otolaryngology Publications

Dept. of Otolaryngology

2017

\title{
Elimination of pain improves specificity of clinical diagnostic criteria for adult chronic rhinosinusitis
}

Scott D. Hirsch

Virginia Commonwealth University

Evan R. Reiter

Virginia Commonwealth University

Laurence J. DiNardo

Virginia Commonwealth University

Wen Wan

University of Chicago

Theodore A. Schuman

Virginia Commonwealth University, theodore.schuman@vcuhealth.org

Follow this and additional works at: http://scholarscompass.vcu.edu/otolar_pubs

Part of the Otolaryngology Commons

(C) 2017 The American Laryngological, Rhinological and Otological Society, Inc.

\section{Downloaded from}

http://scholarscompass.vcu.edu/otolar_pubs/4

This Article is brought to you for free and open access by the Dept. of Otolaryngology at VCU Scholars Compass. It has been accepted for inclusion in Otolaryngology Publications by an authorized administrator of VCU Scholars Compass. For more information, please contact libcompass@vcu.edu. 


\title{
Elimination of Pain Improves Specificity of Clinical Diagnostic Criteria for Adult Chronic Rhinosinusitis
}

\author{
Scott D. Hirsch, BS; Evan R. Reiter, MD, FACS; Laurence J. DiNardo, MD, FACS; \\ Wen Wan, PhD; Theodore A. Schuman, MD
}

\begin{abstract}
Objective: Determine whether the elimination of pain improves accuracy of clinical diagnostic criteria for adult chronic rhinosinusitis.

Study Design: Retrospective cohort study.

Methods: History, symptoms, nasal endoscopy, and computed tomography (CT) results were analyzed for 1,186 adults referred to an academic otolaryngology clinic with presumptive diagnosis of chronic rhinosinusitis. Clinical diagnosis was rendered using the 1997 Rhinosinusitis Taskforce (RSTF) Guidelines and a modified version eliminating facial pain, ear pain, dental pain, and headache.

Results: Four hundred seventy-nine subjects (40\%) met inclusion criteria. Among subjects positive by RSTF guidelines, $45 \%$ lacked objective evidence of sinonasal inflammation by CT, $48 \%$ by endoscopy, and 34\% by either modality. Applying modified RSTF diagnostic criteria, 39\% lacked sinonasal inflammation by CT, 38\% by endoscopy, and 24\% by either modality. Using either abnormal CT or endoscopy as the reference standard, modified diagnostic criteria yielded a statistically significant increase in specificity from $37.1 \%$ to $65.1 \%$, with a nonsignificant decrease in sensitivity from $79.2 \%$ to $70.3 \%$. Analysis of comorbidities revealed temporomandibular joint disorder, chronic cervical pain, depression/anxiety, and psychiatric medication use to be negatively associated with objective inflammation on CT or endoscopy.

Conclusion: Clinical diagnostic criteria overestimate the prevalence of chronic rhinosinusitis. Removing facial pain, ear pain, dental pain, and headache increased specificity without a concordant loss in sensitivity. Given the high prevalence of sinusitis, improved clinical diagnostic criteria may assist primary care providers in more accurately predicting the presence of inflammation, thereby reducing inappropriate antibiotic use or delayed referral for evaluation of primary headache syndromes.
\end{abstract}

Key Words: Chronic sinusitis, facial pain, diagnosis, clinical symptoms, computed tomography.

Level of Evidence: 4.

Laryngoscope, 127:1011-1016, 2017

\section{INTRODUCTION}

Rhinosinusitis is a highly prevalent disease resulting in a considerable burden on the healthcare system. In a 2012 survey, $12 \%$ of U.S. adults reported being diagnosed with rhinosinusitis in the last year, ${ }^{1}$ resulting in more than 20 million annual diagnoses. ${ }^{2}$ A review of ambulatory care data from 2006 to 2010 demonstrated that rhinosinusitis accounted for more antibiotic prescriptions than any other condition, ${ }^{3}$ with $\$ 11$ billion in direct healthcare costs. ${ }^{1,2}$ Further studies have demonstrated a substantial

From the Virginia Commonwealth University School of Medicine (s.D.H.); the Department of Otolaryngology-Head \& Neck Surgery, Virginia Commonwealth University Health System (E.R.R., L.J.D., T.A.S.), Richmond, Virginia; and the Department of Biostatistics, University of Chicago School of Medicine (w.w.), Chicago, Illinois, U.S.A.

Editor's Note: This Manuscript was accepted for publication November 1, 2016.

Poster presentation at the American Rhinologic Society Spring Meeting, Chicago, Illinois, U.S.A., May 5, 2016.

Financial Disclosure: The authors have no funding, financial relationships, or conflicts of interest to disclose.

Send correspondence to Theodore A. Schuman, MD, Department of Otolaryngology-Head \& Neck Surgery, Virginia Commonwealth University School of Medicine, 1200 East Broad Street, Suite 12-313, PO Box 980146 Richmond, VA 23298. E-mail: theodore.schuman@vcuhealth.org

DOI: 10.1002/lary.26442 relationship between acute and chronic rhinosinusitis (CRS) and decreased work productivity, ${ }^{4}$ absenteeism, ${ }^{5}$ and reduced quality of life, suggesting substantial additional indirect costs. ${ }^{6}$

Despite the sizeable impact of rhinosinusitis, considerable variation in diagnosis and management exists across medical specialties. ${ }^{7,8}$ The observed variation in treatment of rhinosinusitis may be due to the fact that gold-standard diagnostic criteria remain elusive, and evaluation in the primary care setting does not have the benefit of nasal endoscopy to gain objective evidence of sinus disease. Furthermore, the correlation between symptoms and objective findings is imperfect.9,10 The prevalence of abnormal sinus computed tomography (CT) in patients reporting symptoms of rhinosinusitis has been reported to be only $65 \%$ to $80 \% .^{9,11-14}$ Numerous conditions may mimic the presentation of rhinosinusitis, including allergic and nonallergic rhinitis, as well as neurologic disorders such as vascular, migraine, or cluster headaches; trigeminal neuralgia; and atypical facial pain.

The 1997 Task Force on Rhinosinusitis (RSTF) defined CRS as the presence of two or more major factors or one major and two minor factors (see Table I) persisting for at least 12 weeks. $^{15}$ The 2016 International Consensus Statement on Rhinosinusitis (ICOR) updated 
TABLE I.

Original and Modified 1997 Rhinosinusitis Task Force Diagnostic Criteria for CRS.

\begin{tabular}{lll}
\hline & \multicolumn{1}{c}{ RSTF } & \multicolumn{1}{c}{ mRSTF } \\
\hline Major Factors & Facial pain/pressure & Facial pressure \\
& Facial congestion/fullness & Facial congestion/fullness \\
& Nasal obstruction/blockage & Nasal obstruction/blockage \\
& Anosmia/hyposmia & Anosmia/hyposmia \\
& Nasal discharge/purulence/discolored & Nasal discharge/purulence/discolored \\
postnasal drainage & postnasal drainage \\
& Purulence in nasal cavity on examination & Purulence in nasal cavity on examination \\
& Fever (acute rhinosinusitis only) & Fever (acute rhinosinusitis only) \\
Headache & Fatigue \\
Hinor Factors & Hatigue & Cough \\
& Halitosis & Fever (all nonacute) \\
& Cough & \\
Dental pain & \\
Ear pain/pressure/fullness & Fever (all nonacute) & \\
\hline \hline
\end{tabular}

CRS = chronic rhinosinusitis; mRSTF = Modified Rhinosinusitis Task Force Criteria; RSTF = Rhinosinusitis Task Force Criteria.

diagnostic criteria for CRS to consist of 12 weeks of at least two of the following symptoms: nasal obstruction/ congestion, anterior/posterior nasal discharge, facial pain/ pressure/fullness, and decreased sense of smell. The 2016 guidelines also added the requirement for objective verification of mucosal inflammation, polyps, and/or purulent sinus drainage via CT or nasal endoscopy for definitive diagnosis of CRS. ${ }^{16}$

Updated diagnostic criteria for CRS continue to include pain as an indicator for sinusitis, although studies have demonstrated poor correlation between facial pain or headache and objective evidence of paranasal sinus inflammation. In rhinologic patients with radiographically or endoscopically confirmed CRS, only $16 \%$ to $20 \%$ complained of facial pain. ${ }^{17-19}$ Similarly, $75 \%$ to $97 \%$ of patients with facial pain attributed to sinus disease lacked radiographic evidence of inflammation on $\mathrm{CT}^{20-22}$ Many patients with facial pain may actually have primary headache, with $88 \%$ of sinus headache sufferers meeting diagnostic criteria for migraine. ${ }^{23}$ Nevertheless, neurologic sources of these symptoms may be mistaken for CRS in the primary care setting. ${ }^{24,25}$

The 2016 ICOR guidelines require the confirmation of symptomatic CRS with objective findings on endoscopy or CT; however, nasal endoscopy is generally unavailable and CT may be underutilized in the primary care setting. In a study by Tan et al., only $50 \%$ of CRS with nasal polyposis (CRSwNP) and $25 \%$ of CRS without nasal polyposis (CRSsNP) subjects underwent CT ordered by the primary care physician (PCP); furthermore, patients were more likely to undergo CT evaluation after initial diagnosis and treatment. $^{26}$ These data suggest that despite updated diagnostic guidelines for CRS requiring CT or nasal endoscopy, many PCPs rely on symptom-based diagnostic criteria that may have high sensitivity but inappropriately low specificity in predicting sinus disease. ${ }^{27,28}$

Given the frequent misattribution of facial pain and headache to sinusitis rather than other potential underlying etiologies, the accuracy of symptom-based clinical criteria for CRS may improve with the elimination of pain, although this does not appear to have been evaluated to date. In this study, we assess the impact on diagnostic accuracy of removal of facial pain and headache from clinical diagnostic criteria for CRS.

\section{MATERIALS AND METHODS}

Billing records were used to identify adult patients referred to the Department of Otolaryngology at Virginia Commonwealth University from November 2008 until June 2015 with a diagnosis of sinusitis. Exclusion criteria included a history of sinonasal surgery, facial trauma, or prior sinus CT, as well as those without subsequent CT or endoscopic evaluation.

Initial otolaryngology encounter notes were reviewed for gender, age, previous rhinosinusitis diagnosis, prior imaging, presenting symptoms, exam findings, medical comorbidities, and current and past medications. Results of nasal endoscopy and sinus CT performed within 3 months of the initial encounter were included. A subsequent diagnosis of a neurologic disorder such as migraine or atypical facial pain was noted.

For each patient, CRS was retrospectively diagnosed by applying the 1997 RSTF diagnostic criteria (see Table I) ${ }^{15} \mathrm{~A}$ modified clinical diagnostic algorithm (mRSTF) was created by removing facial, dental, ear pain, and headache from the symptoms (see Table I). These four symptoms were chosen for elimination because each negatively predicted inflammation on CT or endoscopy in our population. Radiologic presence of mucosal disease was evaluated using the Lund-Mackay scoring system ${ }^{29}$; scores $\geq 3$ were considered indicative of sinusitis. ${ }^{11,30}$ Endoscopic disease burden was rated using the Modified Lund-Kennedy (MLK) scoring system, ${ }^{31}$ with scores $\geq 2$ considered positive for sinusitis.

Clinical and demographic characteristics were summarized by descriptive statistics. Sensitivity, specificity, accuracy, positive predictive value (PPV), and negative predictive value (NPV) were calculated for RSTF and mRSTF criteria using either CT or endoscopy as the objective standard for sinusitis, as per the 2016 ICOR guidelines. A binomial z-test was used to compare RSTF and mRSTF in sensitivity, specificity, accuracy, 
TABLE II.

Relationship Between Presenting Symptoms and Objective Evidence of Inflammation Using Either CT or Nasal Endoscopy as the Reference Standard.

\begin{tabular}{|c|c|c|c|c|c|c|}
\hline Symptom & $\mathrm{N}$ & $\begin{array}{l}\text { Regression } \\
\text { Coefficient }\end{array}$ & $\begin{array}{l}\text { Standard } \\
\text { Error }\end{array}$ & $\begin{array}{l}\text { Odds } \\
\text { Ratio }\end{array}$ & $95 \% \mathrm{Cl}$ & $P$ Value \\
\hline Facial pain & 84 & -.652 & 0.196 & 0.52 & (0.35 to 0.77$)$ & $0.001^{*}$ \\
\hline Facial pressure & 120 & 0.352 & 0.196 & 1.42 & (0.97 to 2.09 ) & 0.073 \\
\hline Nasal obstruction & 217 & 1.157 & 0.198 & 3.18 & (2.16 to 4.69 ) & $0.000^{*}$ \\
\hline Purulent rhinorrhea & 153 & 1.173 & 0.205 & 3.23 & (2.16 to 4.83 ) & $0.000^{*}$ \\
\hline Anosmia/hyposmia & 74 & 1.278 & 0.294 & 3.59 & (2.02 to 6.39 ) & $0.000^{*}$ \\
\hline Headache & 130 & -.574 & 0.190 & 0.56 & (0.39 to 0.82$)$ & $0.002^{*}$ \\
\hline Fatigue & 32 & -.282 & 0.282 & 0.75 & (0.43 to 1.31$)$ & 0.319 \\
\hline Dental pain & 14 & -.759 & 0.369 & 0.47 & (0.23 to 0.97$)$ & $0.040^{*}$ \\
\hline Ear pressure/pain & 52 & -.533 & 0.225 & 0.59 & (0.38 to 0.91$)$ & $0.018^{*}$ \\
\hline Cough & 66 & 0.374 & 0.241 & 1.45 & (0.91 to 2.33 ) & 0.121 \\
\hline Halitosis & 15 & -.153 & 0.409 & 0.86 & (0.39 to 1.91$)$ & 0.709 \\
\hline Fever & 12 & 0.663 & 0.585 & 1.94 & (0.62 to 6.11$)$ & 0.257 \\
\hline
\end{tabular}

*Signifies statistical significance.

$\mathrm{Cl}=$ confidence interval; $\mathrm{CT}=$ computed tomography; $\mathrm{N}=$ number of subjects.

PPV, and NPV. Somers' D was used to measure the concordant rate between clinical diagnostic criteria and CT. Logistic regression was used to test association of symptoms with CT and endoscopy scores. Forest plots via a logistic regression were used to illustrate the associations of comorbid conditions with diagnostic results. Significance was determined using the type I error of 5\%. Statistical analysis was performed using SAS software version 9.4 (SAS Institute Inc., Cary, NC). This study was reviewed and approved by the Institutional Review Board of Virginia Commonwealth University.

\section{RESULTS}

The query of billing records identified 1,186 potential subjects; of those, 479 (40.4\%) met inclusion criteria. There were $317(66.2 \%)$ females and $162(33.8 \%)$ males; with mean age 50.3 years (standard deviation $(\mathrm{SD}) \pm 15.1$, range $19-89$ ). Utilizing RSTF criteria, 349 (72.9\%) met criteria for diagnosis of CRS, versus $271(56.6 \%)$ using mRSTF criteria.

Among subjects, 396 (82.7\%) underwent CT after initial otolaryngology evaluation, with average LundMackay score 5.1 (SD \pm 5.6 , range $0-24$, median 3 ), and $203(51.3 \%)$ with Lund-Mackay > 3, meeting criteria for sinusitis. There were $388(81.0 \%)$ subjects with nasal endoscopy performed at the initial encounter, with mean MLK score 1.6 (SD \pm 2.0 , range $0-10$, median 1 ) and 179 (46.1\%) with MLK > 2, meeting criteria for sinusitis. There were $293(61.2 \%)$ subjects who fulfilled either CT or endoscopic criteria for sinusitis, and 101 (21.1\%) who fulfilled both CT and endoscopic criteria. Concordance between CT and endoscopy for diagnosing sinusitis was fair, with a Kappa coefficient of 0.302 (95\% confidence interval [CI] 0.196-0.407, $P<0.0001)$. The frequency of symptoms and their associations with objective evidence of sinusitis is presented in Table II.

Of those subjects with RSTF diagnosis of CRS, 54.8\% had CT evidence, $52.0 \%$ had endoscopic evidence, and $66.5 \%$ had either CT or endoscopic evidence of sinusitis. Of those who met mRSTF criteria, $60.6 \%$ had CT evidence, $61.6 \%$ had endoscopic evidence, and $76.0 \%$ had either CT or endoscopic evidence of sinusitis. The concordance between either positive CT or endoscopy and RSTF diagnosis was $29.4 \%$, with Somers' D of $0.163(P<0.0001)$, whereas the concordance between either CT or endoscopy and mRSTF was $45.7 \%$, with Somers' D of $0.354(P<$ 0.0001). Sensitivity, specificity, and accuracy of both diagnostic criteria were evaluated using abnormal CT, abnormal endoscopy, or either abnormal CT or endoscopy as the reference standard for diagnosis of sinusitis (see Table III). Use of mRSTF criteria resulted in a large increase in specificity $(P<0.05)$ but correspondingly small, nonsignificant trend toward decrease in sensitivity.

Comorbidities were analyzed for association with CT or endoscopic findings. Among our subjects, $4.4 \%$ had fibromyalgia; $6.1 \%$ had neuropathy; $11.1 \%$ had chronic cervical pain; $14.4 \%$ had a history of migraine; $7.3 \%$ had temporomandibular joint disorder (TMD); $18.8 \%$ had a history of depression or anxiety; and $33.9 \%$ reported a history of psychiatric medication use. Subjects with any one of these comorbidities were less likely to have sinusitis on either CT or endoscopy (relative risk $[\mathrm{RR}]=0.69$, 95\% CI 0.578-0.828, $P<0.0001)$. Individually, a history of chronic cervical pain $(\mathrm{RR}=0.62,95 \%$ CI $0.442-0.872$, $P=0.0006)$, TMD $(\mathrm{RR}=0.54,95 \%$ CI $0.341-0.862, P=$ $0.0007)$, depression/anxiety $(\mathrm{RR}=0.81,95 \%$ CI $0.650-$ $1.00, P=0.031$ ), and prior use of psychiatric medications $(\mathrm{RR}=0.85,95 \%$ CI $0.724-1.00, P=0.047)$ were inversely associated with the presence of sinusitis on either CT or endoscopy (see Fig. 1).

\section{DISCUSSION}

Prior studies have shown poor correlation between symptoms and endoscopic or radiographic evidence of CRS. ${ }^{14,32}$ Updated 2016 ICOR diagnostic criteria emphasized the need for objective evidence of sinonasal inflammation to confirm the diagnosis of CRS. Whereas this requirement is readily applicable to practicing otolaryngologists, endoscopy is not available and CT may be underused 
TABLE III.

Sensitivity, Specificity, Accuracy, PPV, and NPV of Clinical and Modified Clinical Diagnostic Criteria for CRS Using CT, Nasal Endoscopy, and CT or Nasal Endoscopy as the Reference Standard.

\begin{tabular}{llcccccc}
\hline \multicolumn{2}{c}{ Epidemiology Data } & N & Sensitivity (\%) & Specificity (\%) & Accuracy (\%) & PPV (\%) & NPV (\%) \\
\hline CT & RSTF & 161 & 79.3 & 31.1 & 55.8 & 54.8 & 58.8 \\
& mRSTF & 143 & 70.4 & 51.8 & 61.4 & 60.6 & 62.5 \\
& Difference & 18 & -8.9 & +20.7 & +5.6 & +5.8 & +3.7 \\
& $P$ value & - & 0.181 & $0.030^{*}$ & 0.301 & 0.292 & 0.418 \\
Endoscopy & RSTF & 146 & 81.6 & 35.4 & 56.7 & 52.0 & 69.2 \\
& mRSTF & 135 & 75.4 & 59.8 & 67.0 & 71.6 & 74.0 \\
& Difference & 11 & -6.2 & +24.4 & +10.3 & +9.6 & +4.8 \\
& P value & - & 0.410 & 0.063 & 0.265 & 0.285 & 0.484 \\
CT or Endoscopy & RSTF & 245 & 79.2 & 37.1 & 62.8 & 66.5 & 53.1 \\
& mRSTF & 215 & 70.3 & 65.1 & 68.3 & 76.0 & 58.2 \\
& Difference & 30 & -8.9 & +28 & +5.5 & +9.5 & +5.1 \\
& P value & - & 0.082 & $0.0003^{*}$ & 0.179 & 0.072 \\
\hline
\end{tabular}

$P$ values calculated using one-sided binomial test.

*Denotes statistical significance.

$\mathrm{CT}=$ computed tomography; mRSTF = Modified Rhinosinusitis Task Force Criteria; $\mathrm{N}=$ number of subjects; NPV $=$ negative predictive value; PPV = positive predictive value; RSTF $=$ Rhinosinusitis Task Force Criteria

by PCPs. ${ }^{26}$ Clinical diagnostic criteria established by the 1997 RSTF and updated in the 2016 ICOR statement both include pain as a symptom contributing to the diagnosis of CRS. Although facial pain or headache can indeed be due to CRS, several studies have established that facial pain and sinus headache are frequently migrainous or otherwise neurologic in etiology. ${ }^{33-36} \mathrm{~A}$ reliance on symptom-based diagnosis, as is likely the norm in the primary care setting, may lead to frequent misattribution of neurologic facial pain to CRS, leading to the inappropriate prescription of antibiotics and delay in management of underlying pathology.

The current study seeks to determine whether elimination of pain-related symptoms could improve clinical accuracy for diagnosis of CRS via symptom-based criteria. The 1997 RSTF criteria were chosen for analysis

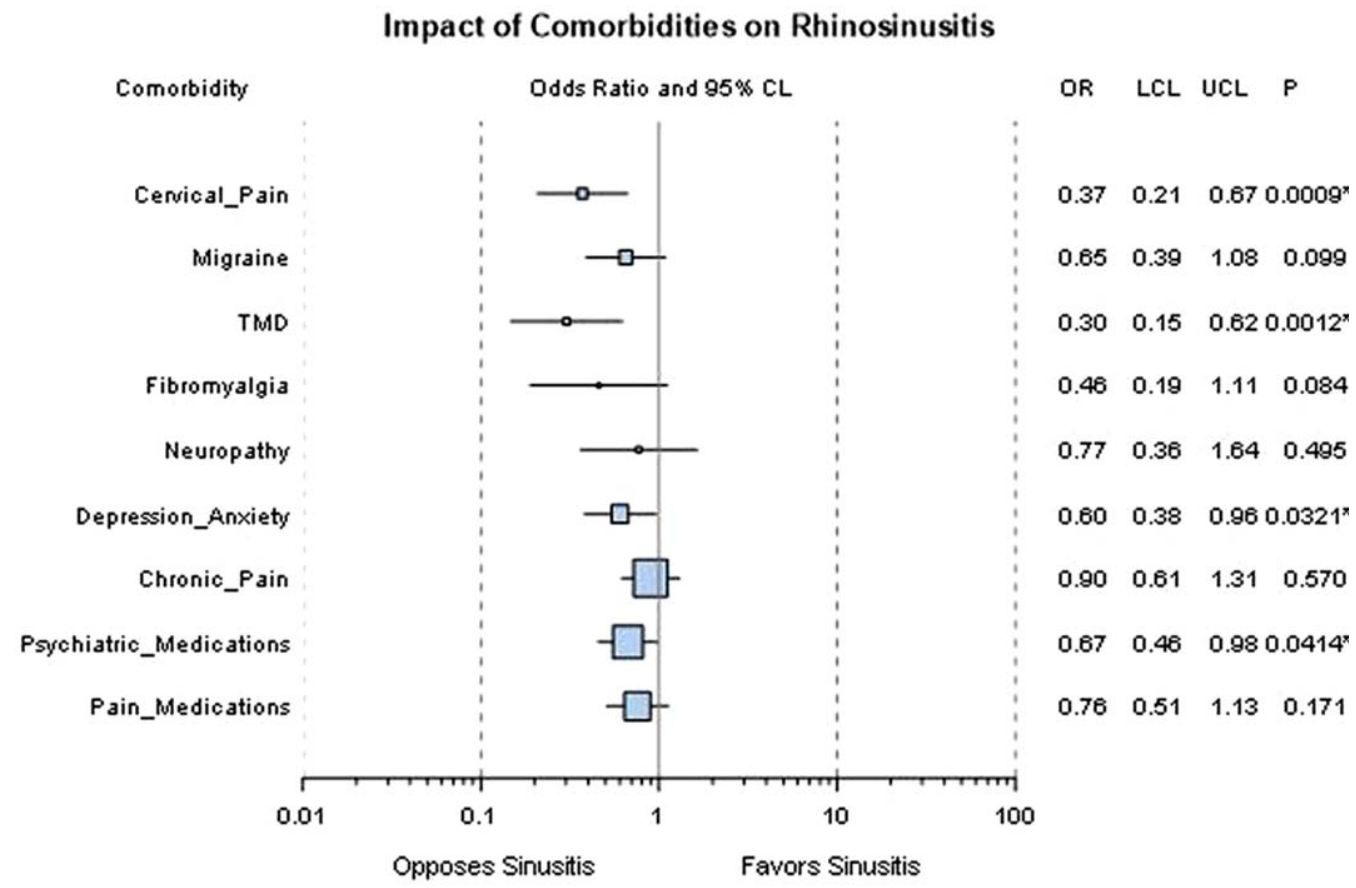

Fig. 1. Forest plot demonstrating impact of individual comorbid conditions on the diagnosis of rhinosinusitis using either computed tomography or nasal endoscopy as the reference standard.

Box sizes represent the prevalence of symptoms.

${ }^{\star}$ Denotes statistical significance.

$\mathrm{LCL}=$ lower confidence limit; $\mathrm{OR}=$ odds ratio; TMD = temporomandibular joint disease; UCL = upper confidence limit. [Color figure can be viewed in the online issue, which is available at www.laryngoscope.com.] 
because these guidelines do not require objective confirmation of sinonasal inflammation, and thus may better represent the diagnostic process employed by PCPs. When applied retroactively to our cohort, mRSTF criteria resulted in a significant increase in specificity without a concordant loss of sensitivity in the diagnosis of CRS. There was an upward trend in overall diagnostic accuracy for mRSTF criteria, regardless of whether CT, endoscopy, or either CT or endoscopy were used as the reference standard for sinonasal inflammation.

Applying traditional RSTF criteria to our data demonstrated a PPV of $54.8 \%$ for abnormal CT, similar to published data. ${ }^{10}$ This low PPV portends frequent falsepositive clinical diagnoses of sinusitis, supporting the notion that symptom-based diagnostic criteria are poorly suited to differentiating CRS from other diseases that may manifest similar symptoms. ${ }^{37-39}$ Use of mRSTF criteria led to no statistical change in PPV or NPV, although both trended higher. Despite more stringent criteria, the reduction of false positive diagnoses seen with the elimination of headache and pain from the mRSTF criteria did not result in a significant loss of sensitivity or NPV within our study population.

Prior studies have demonstrated that nasal obstruction, ${ }^{40-43}$ postnasal drip, ${ }^{42}$ purulent rhinorrhea, ${ }^{41,43}$ and smell disturbances ${ }^{42-45}$ are associated with the presence of sinonasal inflammation. Likewise, the current study demonstrated that subjects with purulent rhinorrhea, nasal obstruction, and anosmia were more likely to have abnormal CT or endoscopy, and that patients with headache, facial, dental, and ear pain were more likely to have normal radiographic or endoscopy.

Comorbidities characterized by chronic head and neck pain, particularly cervical pain and TMD, negatively predicted the presence of sinusitis on CT or endoscopy within this study population. Migraines and fibromyalgia both narrowly missed statistical significance as negative predictors of sinus disease. Additionally, patients with a history of depression/anxiety and psychiatric medication use were less likely to have objection inflammation. These findings suggest that subjects with comorbidities characterized by chronic head and neck pain or depression/anxiety may have lower pretest probabilities of CRS, and thus benefit from earlier CT and neurologic evaluation, rather than trials of extended antimicrobial therapy. Prior work by Tan et al. supports the notion that upfront CT may be advantageous over empiric antibiotic therapy in the primary care setting. ${ }^{46-48}$

Rhinosinusitis is a highly prevalent disease resulting in considerable personal and societal costs. ${ }^{1,3-6}$ Although 20 million diagnoses of sinusitis are made in the United States annually, a growing body of research indicates that accurate diagnosis of sinusitis, especially using symptom-based criteria, is problematic. Use of objective modalities such as CT or nasal endoscopy may improve diagnostic accuracy, but are underused or unavailable in the primary care setting where many initial diagnoses of CRS are made. Moreover, upfront CT has been suggested to be more cost-efficient in most circumstances when compared to empiric antibiosis, ${ }^{46,47}$ but if overused may produce an unacceptable increase in the incidental finding of mucosal thickening and unnecessary treatment of presumed sinusitis. ${ }^{49}$ An improved ability to parse out symptomatic sinonasal inflammation is critical in optimizing diagnosis of CRS. In this study, the elimination of headache, ear, dental, and facial pain from clinical criteria significantly improved specificity without sacrificing sensitivity, and may be applicable to a broader population undergoing evaluation by PCPs for possible CRS.

This study is limited by selection bias, in that our population consisted of patients with suspected CRS referred to an academic otolaryngology practice. The presence of a comparison group without symptoms of CRS would help control for confounding variables and demonstrate baseline characteristics in a healthy population. Additionally, the retrospective design and lack of standardized questionnaires or endoscopic rating systems may have reduced the reliability of data extracted from the study population. Broad, poorly defined symptoms such as pain may be particularly difficult to operationalize when performing a retrospective review. ${ }^{50}$ In the absence of a structured method for querying symptoms, patients might focus on more tangible symptoms of CRS, such as obstruction or drainage, leading to an underrepresentation of facial pain/pressure in participants with legitimate inflammatory disease.

Gender is another potential source of bias because women constituted $66.2 \%$ of the study population and are more likely to report facial pain and headache. ${ }^{51} \mathrm{Lal}$ et al demonstrated that women presented with higher Sino-Nasal Outcome Test 22 and lower Lund-Mackay scores than men, ${ }^{52}$ indicating a tendency to report more severe sinonasal symptoms despite lesser objective evidence of inflammation. An over-representation of women within this cohort could result in a misleadingly low specificity for diagnostic criteria. However, gender subgroup analysis showed minimal effect. For the entire cohort, using abnormal CT or endoscopy as the reference standard, the specificity and sensitivity of the mRSTF criteria were $65.1 \%$ and $70.3 \%$, respectively, whereas these changed to $68.2 \%$ and $67.8 \%$ for male subjects, and to $64.1 \%$ and $72.0 \%$ for female subjects, respectively.

This study was designed to assess diagnostic accuracy for CRS using clinical criteria in previously undiagnosed patients, as may frequently present to PCPs. Patients with a history of sinonasal surgery were excluded because one might reasonably expect that a positive CT or endoscopy led to prior surgical intervention. Excluding patients with previous surgery could bias the population to less significant disease, impacting sensitivity or specificity calculations. Additionally, the predictive value of pain for true inflammatory disease could potentially differ in the postoperative population.

Not all study participants had both CT and nasal endoscopy performed. Given the imperfect concordance between these modalities found among subjects who underwent both procedures, it is possible that patients with only a negative CT or endoscopy were considered not to have sinusitis; although if performed, a second test may have been positive. This would result in an inappropriately low estimation of sensitivity within the 
study population; however, we would expect that this effect would alter specificity and sensitivity calculations for both RSTF and mRSTF diagnostic criteria in parallel fashion, and thus not likely impact the nature of our conclusions. Future prospective studies could employ validated questionnaires and endoscopy-scoring systems to verify whether the trends identified in this retrospective cohort can be generalized to a greater population with presumed CRS.

\section{CONCLUSION}

The elimination of facial pain, dental pain, ear pain, and headache from clinical diagnostic criteria for CRS resulted in significantly improved specificity for diagnosis of sinusitis as confirmed via CT or nasal endoscopy. Ongoing research is necessary to optimize diagnostic algorithms for CRS, particularly in the primary care setting, which may in turn lead to decreased inappropriate antibiotic use and delay in management of primary headache syndromes.

\section{BIBLIOGRAPHY}

1. Blackwell DL, Lucas JW, Clarke TC. Summary health statistics for U.S. adults: national health interview survey, 2012. Vital Health Stat 10 2014;260:1-161.

2. Anon JB, Jacobs, MR, Poole MD, et al. Antimicrobial treatment guidelines for acute bacterial rhinosinusitis. Otolaryngol Head Neck Surg 2004; 130(suppl):1-45.

3. Smith SS, Evans CT, Tan BK., Chandra RK, Smith SB, Kern RC. National burden of antibiotic use for adult rhinosinusitis. J Allergy Clin Immunol 2013;132:1230-1232

4. Stankiewicz J, Tami T, Truitt T, et al. Impact of chronic rhinosinusitis on work productivity through one-year follow-up after balloon dilation of the ethmoid infundibulum. Int Forum Allergy Rhinol 2011;1:38-45.

5. Bhattacharyya N, Grebner J, Martinson NG. Recurrent acute rhinosinusitis: epidemiology and health care cost burden. Otolaryngol Head Neck Surg 2012;146:307-312.

6. Gliklich RE, Metson R. The health impact of chronic sinusitis in patients seeking otolaryngologic care. Otolaryngol Head Neck Surg 1995;113: 104-109.

7. Kaszuba SM, Stewart MG. Medical management and diagnosis of chronic rhinosinusitis: a survey of treatment patterns by United States otolaryngologists. Am J Rhinol 2006;20:186-190.

8. Winstead W. Rhinosinusitis. Prim Care 2003;30:137-154

9. Bhattacharyya T, Piccirillo J, Wippold FJ. Relationship between patientbased descriptions of sinusitis and paranasal sinus computed tomographic findings. Arch Otolaryngol Head Neck Surg 1997;123:1189-1192.

10. Hwang P, Irwin S, Griest S, Caro J, Nesbit G. Radiologic correlates of symptom-based diagnostic criteria for chronic rhinosinusitis. Otolaryngol Head Neck Surg 2003;128:489-496.

11. Agius AM. Chronic sinusitis in Malta-correlation between symptoms and CT scan. Rhinology 2010;48:59-64.

12. Conley D, Pearlman A, Zhou, K, Chandra R, Kern R. The role of point-ofcare CT in the management of chronic rhinosinusitis: a case-control study. Ear Nose Throat J 2011;90:376-381.

13. Stewart MG, Johnson RF. Chronic sinusitis: symptoms versus CT scan findings. Curr Opin Otolaryngol Head Neck Surg 2004;12:27-29.

14. Hwang PH, Irwin SB, Griest SE, Caro JE, Nesbit GM. Radiologic correlates of symptom-based diagnostic criteria for chronic rhinosinusitis. Otolaryngol Head Neck Surg 2003;128:489-496.

15. Lanza DC, Kennedy DW. Adult rhinosinusitis defined. Otolaryngol Head Neck Surg 1997;117:S1-S7.

16. Orlandi RR, Kingdom TT, Hwang PH, et al. International consensus statement on allergy and rhinology: rhinosinusitis. Int Forum Allergy Rhinol 2016;6(suppl 1):S22-S209.

17. Clifton NJ, Jones NS. Prevalence of facial pain in 108 consecutive patients with paranasal mucopurulent discharge at endoscopy. J Laryngol Otol 2007; $121: 345-348$

18. Fahy C, Jones NS. Nasal polyposis and facial pain. Clin Otolaryngol Allied Sci 2001;26:510-513.

19. Eweiss AZ, Lund VJ, Barlow J, Rose G. Do patients with chronic rhinosinusitis with nasal polyps suffer with facial pain. Rhinology 2013;51: 231-235.

20. Foroughipour M, Sharifian SM, Shoeibi A, Ebdali Barabad N, Bakhshaee M. Causes of headache in patients with a primary diagnosis of sinus headache. Eur Arch Otorhinolaryngol 2011;268:1593-1596.
21. Eross E, Dodick D, Eross M. The Sinus, Allergy and Migraine Study (SAMS). Headache 2007;47:213-224.

22. West B, Jones NS. Endoscopy-negative, computed tomography-negative facial pain in a nasal clinic. Laryngoscope 2001;111:581-586.

23. Schreiber CP, Hutchinson S, Webster CJ, Ames M, Richardson MS, Powers C. Prevalence of migraine in patients with a history of self-reported or physiciandiagnosed "sinus" headache. Arch Intern Med 2004;164:1769-1772.

24. Agius AM, Jones NS, Muscat R. Prospective three-year follow up of a cohort study of 240 patients with chronic facial pain. $J$ Laryngol Otol 2014;128:518-526.

25. Jones NS. The prevalence of facial pain and purulent sinusitis. Curr Opin Otolaryngol Head Neck Surg 2009;17:38-42.

26. Tan B K, Chandra RK, Pollak J, et al. Incidence and associated premorbid diagnoses of patients with chronic rhinosinusitis. J Allergy Clin Immunol 2013;131:1350-1360.

27. Hsueh WD, Conley DB, Kim H, et al. Identifying clinical symptoms for improving the symptomatic diagnosis of chronic rhinosinusitis. Int Forum Allergy Rhinol 2013;3:307-314.

28. Pynnonen M, Fowler K, Terrell JE. Clinical predictors of chronic rhinosinusitis. Am J Rhinol 2007;21:159-163.

29. Lund VJ, Kennedy DW. Staging for rhinosinusitis. Otolaryngol Head Neck Surg 1997;117:S35-S40.

30. Bhattacharyya N, Fried MP. The accuracy of computed tomography in the diagnosis of chronic rhinosinusitis. Laryngoscope 2003;113:125-129.

31. Psaltis AJ, Li G, Vaezeafshar R, Cho KS, Hwang PH. Modification of the Lund-Kennedy endoscopic scoring system improves its reliability and correlation with patient-reported outcome measures. Laryngoscope 2014; 124:2216-2223.

32. Kennedy DW. The overall relationship between CT imaging and symptom scores in chronic rhinosinusitis (CRS). Int Forum Allergy Rhinol 2013;3: 951-952.

33. Cashman EC, Smyth D. Primary headache syndromes and sinus headache: an approach to diagnosis and management. Auris Nasus Larynx 2012:39:257-260.

34. Lal D, Rounds A, Dodick DW. Comprehensive management of patients presenting to the otolaryngologist for sinus pressure, pain, or headache. Laryngoscope 2015; 125:303-310.

35. Lal D, Rounds AB, Rank MA, Divekar R. Clinical and 22-item Sino-Nasal Outcome Test symptom patterns in primary headache disorder patients presenting to otolaryngologists with "sinus" headaches, pain or pressure. Int Forum Allergy Rhinol 2015;5:408-416.

36. Mehle ME, Schreiber CP. Sinus headache, migraine, and the otolaryngologist. Otolaryngol Head Neck Surg 2005;133:489-496.

37. Harar RP, Chadha NK, Rogers G. The role of septal deviation in adult chronic rhinosinusitis: a study of 500 patients. Rhinology 2004;42:126-130.

38. Katle EJ, Hatlebakk JG, Steinsvag S. Gastroesophageal reflux and rhinosinusitis. Curr Allergy Asthma Rep 2013;13:218-223.

39. Orlandi RR. A systematic analysis of septal deviation associated with rhinosinusitis. Laryngoscope 2010;120:1687-1695.

40. Abrass LJ, Chandra RK, Conley DB, Tan BK, Kern RC. Factors associated with computed tomography status in patients presenting with a history of chronic rhinosinusitis. Int Forum Allergy Rhinol 2011;1:178-182.

41. Leo G, Incorvaia C, Cazzavillan A, Consonni D. May chronic rhinosinusitis in children be diagnosed by clinical symptoms. Int $J$ Pediatr Otorhinolaryngol 2015;79:825-828.

42. Park DY, Lee EJ, Kim JH, Kim YS, Jung CM, Kim KS. Correlation between symptoms and objective findings may improve the symptombased diagnosis of chronic rhinosinusitis for primary care and epidemiological studies. BMJ Open 2015;5:e009541.

43. Dietz de Loos DA, Hopkins C, Fokkens WJ. Symptoms in chronic rhinosinusitis with and without nasal polyps. Laryngoscope 2013;123:57-63.

44. Bonfils P, Halimi P, Le Bihan C, Nores JM, Avan P, Landais P. Correlation between nasosinusal symptoms and topographic diagnosis in chronic rhinosinusitis. Ann Otol Rhinol Laryngol 2005;114:74-83.

45. Bhattacharyya, N. Clinical and symptom criteria for the accurate diagnosis of chronic rhinosinusitis. Laryngoscope 2006;116:1-22.

46. Leung R, Kern R, Jordan N, et al. Upfront computed tomography scanning is more cost-beneficial than empiric medical therapy in the initial management of chronic rhinosinusitis. Int Forum Allergy Rhinol 2011;1: 471-480.

47. Leung RM, Chandra RK, Kern RC, Conley DB Tan BK. Primary care and upfront computed tomography scanning in the diagnosis of chronic rhinosinusitis: a cost-based decision analysis. Laryngoscope 2014;124:12-18.

48. Tan BK, Chandra RK, Conley DB, Tudor RS, Kern RC. A randomized trial examining the effect of pretreatment point-of-care computed tomography imaging on the management of patients with chronic rhinosinusitis symptoms. Int Forum Allergy Rhinol 2011;1:229-234.

49. Jones NS. CT of the paranasal sinuses: a review of the correlation with clinical, surgical and histopathological findings. Clin Otolaryngol Allied Sci 2002;27:11-17.

50. Vassar M, Holzmann M. The retrospective chart review: important methodological considerations. J Educ Eval Health Prof 2013;10, 12.

51. Busaba NY, Sin HJ, Salman SD. Impact of gender on clinical presentation of chronic rhinosinusitis with and without polyposis. J Laryngol Otol 2008; $122: 1180-1184$

52. Lal D, Rounds AB, Divekar R. Gender-specific differences in chronic rhinosinusitis patients electing endoscopic sinus surgery. Int Forum Allergy Rhinol 2016;6:278-286. 